\title{
An Unusual Presentation of Prostate Carcinoma
}

\begin{abstract}
Prostate carcinoma is the second most common cancer among men worldwide. Although prostate carcinoma is common, its presentation resembling retroperitoneal fibrosis is uncommon. We report a patient with prostate carcinoma mimicking retroperitoneal fibrosis. An elderly male presenting in a volume overload state with features of obstructive uropathy was diagnosed as a case of prostate carcinoma. Magnetic resonance imaging was suggestive of retroperitoneal fibrosis. The presentation of prostate carcinoma as retroperitoneal fibrosis is rare.
\end{abstract}

Keywords: Obstructive uropathy, prostate carcinoma, retroperitoneal fibrosis

\section{Introduction}

Retroperitoneal fibrosis is characterized by the extensive development of inflammatory fibrotic tissue in the retroperitoneum, leading to the compression and obstruction of the ureters and other adjacent organs. We report a patient with obstructive uropathy due to retroperitoneal fibrosis, diagnosed as a case of prostate carcinoma.

\section{Case Report}

A 67-year-old male presented bilateral pedal edema for 1 month, scrotal edema, and backache and reduced urine output for 15 days. He was a smoker, alcoholic, and a hypertensive for the past 6 years on irregular treatment. Examination showed pallor, bilateral pitting pedal edema, scrotal swelling, and edematous external genitalia. Per-rectal examination revealed hard prostate Grade II.

Complete blood count showed hemoglobin of $9 \mathrm{~g} / \mathrm{dl}$. Blood urea and serum creatinine were $135 \mathrm{mg} / \mathrm{dl}$ and $3.9 \mathrm{mg} / \mathrm{dl}$, respectively. Serum sodium and potassium were $139 \mathrm{mEq} / \mathrm{L}$ and $5.1 \mathrm{mEq} / \mathrm{L}$, respectively. Serum uric acid was $12 \mathrm{mg} / \mathrm{dl}$. Liver function test, blood sugar, lipid profile, serum calcium, and phosphorous were normal. Serum protein electrophoresis, electrocardiogram, chest radiograph, and echocardiogram were normal. Ultrasound abdomen and pelvis showed bilateral hydroureteronephrosis.

This is an open access journal, and articles are distributed under the terms of the Creative Commons Attribution-NonCommercial-ShareAlike 4.0 License, which allows others to remix, tweak, and build upon the work non-commercially, as long as appropriate credit is given and the new creations are licensed under the identical terms.

For reprints contact: reprints@medknow.com
Plain computed tomography of the kidneys, ureters, and bladder (CT KUB) showed well-defined isodense circumferential thickening around the abdominal aorta below the level of superior mesenteric artery till bifurcation, bilateral hydroureteronephrosis, and isodense areas in the prevertebral region at level of L1, $\mathrm{S} 5$, features suggestive of retroperitoneal fibrosis [Figure 1].

Magnetic resonance imaging (MRI) abdomen and pelvis showed the medial deviation of ureters, multiple bone metastases and enlarged prostate, features consistent with retroperitoneal fibrosis, and metastatic prostatic malignancy [Figure 2]. Antinuclear antibody test was negative. Serum alkaline phosphatase was 267 IU/L. Prostate-specific antigen was found to be $905.6 \mathrm{ng} / \mathrm{ml}$.

Trucut biopsy of the prostate was done which confirmed prostate adenocarcinoma, Gleason score 6/10 [Figure 3]. As the patient was not willing for any surgical intervention, he was treated conservatively.

\section{Discussion}

Retroperitoneal fibrosis is an uncommon fibrotic reaction in the retroperitoneum that typically presents with ureteral obstruction. ${ }^{[1]}$ Incidence is 1.38 cases per 100,000 , and the mean age is approximately 64 years with male-to-female ratio of $3: 1 .^{[2]}$ Etiology includes idiopathic or Ormond's disease (70\%); radiation; medications such as hydralazine and beta-blockers;

How to cite this article: Sadasivan G, Arul R, Prabaharan A. An unusual presentation of prostate carcinoma. Indian J Med Paediatr Oncol 2018;39:521-3.

\section{Greeshma}

Sadasivan, R. Arul ${ }^{1}$, A. Prabaharan ${ }^{1}$

Departments of General Medicine and ${ }^{l}$ Nephrology, Coimbatore Medical College Hospital, Coimbatore,

Tamil Nadu, India
Address for correspondence: Dr. Greeshma Sadasivan, B.N. 439, Bapuji Nagar, Medical College, P.O.,

Thiruvananthapuram - 695 011, Kerala, India.

E-mail: gladeyepansyi@ yahoo.in

Access this article online

Website: www.ijmpo.org

DOI: 10.4103/ijmpo.ijmpo_37_17 Quick Response Code:

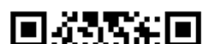
Q $45 \mathrm{H}$

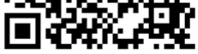




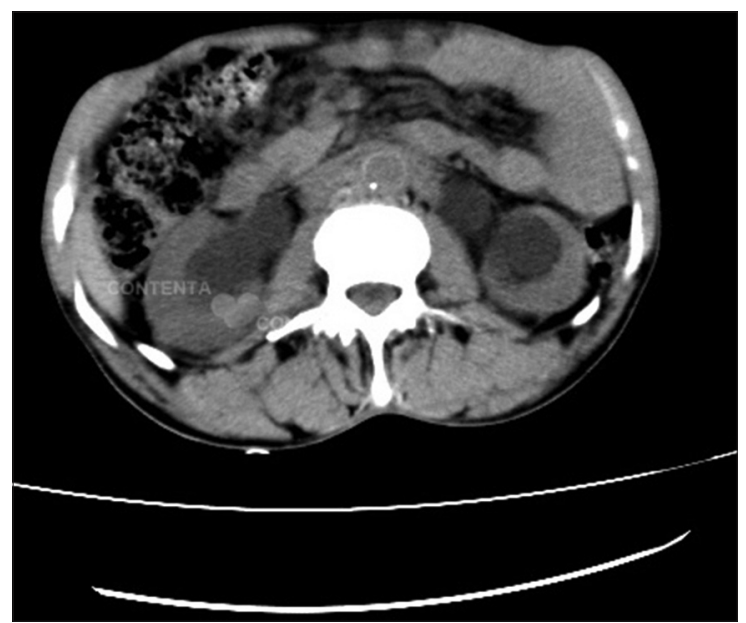

Figure 1: Plain computed tomography of kidneys, ureters, and bladder showing well-defined isodense circumferential thickening around the abdominal aorta below the level of superior mesenteric artery till bifurcation, bilateral hydroureteronephrosis, isodense areas noted in prevertebral region at level of L1, S5, features suggestive of retroperitoneal fibrosis

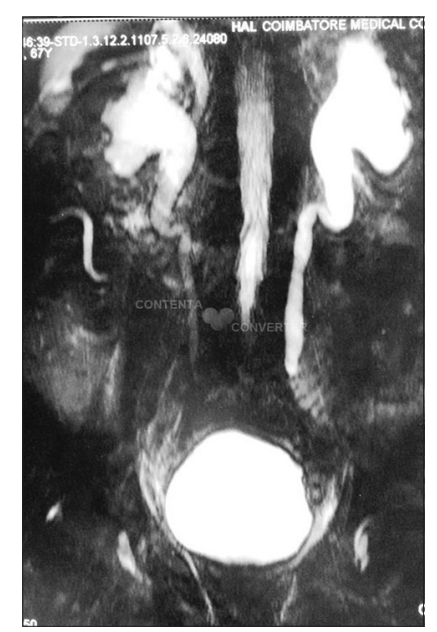

Figure 2: Magnetic resonance imaging abdomen and pelvis showing medial deviation of ureters, multiple bone metastases and enlarged prostate, features consistent with retroperitoneal fibrosis, and metastatic prostatic malignancy

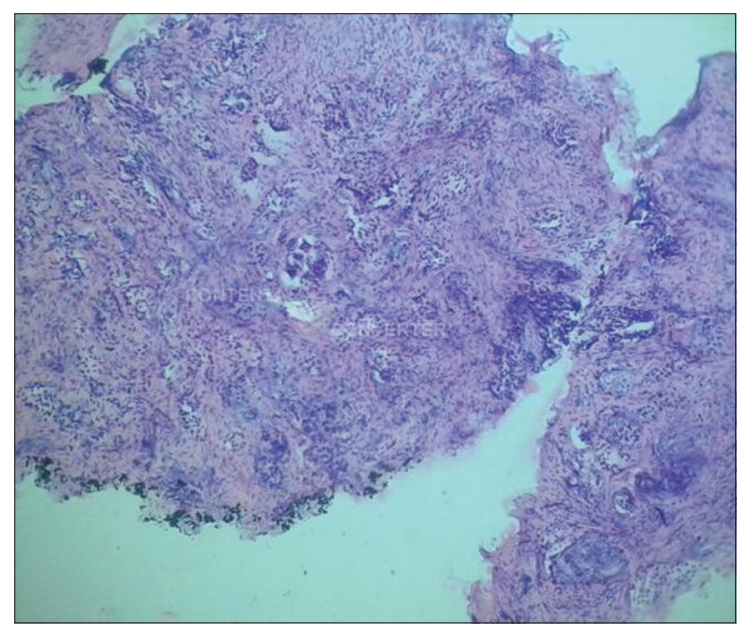

Figure 3: Histopathological analysis of the lesion showing prostate adenocarcinoma, Gleason score 6/10 inflammation such as pancreatitis and pyelonephritis; malignancies such as prostate, breast, and lymphoma carcinoma; asbestos exposure; and retroperitoneal bleeding. ${ }^{[3,4]}$

Prostate carcinoma is the second most common cancer and cancer-related deaths among men worldwide. It is primarily a disease of an elderly male. In the United States, approximately 200,000 new cases are diagnosed each year and $95 \%$ are adenocarcinomas. ${ }^{[5]}$ It spreads by local invasion, lymphatic spread, or by hematogenous metastases.

Fibrosis begins around the aorta. Thinning of the media allows insoluble lipids such as ceroid, to leak into periaortic tissue, stimulating an immunologic reaction leading to fibrosis. Drugs such as beta-blockers may act as haptens leading to autoimmune reaction.

Signs and symptoms of retroperitoneal fibrosis are nonspecific. ${ }^{[6]}$ The most common symptom is poorly localized pain in the flank, back, and scrotum. Others include anemia, lower extremity edema, and urinary frequency. ${ }^{[3,7,8]}$ Our patient also presented with backache.

Most cases of prostate cancer are identified by screening in asymptomatic men. Symptoms occur only when locally advanced or metastatic and include urinary complaints and back pain. ${ }^{[9]}$

$\mathrm{CT}$ in retroperitoneal fibrosis shows periaortic soft-tissue mass that envelops aorta and inferior vena cava between the renal hila and the sacral promontory. ${ }^{[2,3,8]}$ Pelvic extension and medial deviation of the ureters are common features in MRI. ${ }^{[3,10]}$

CT scan detects enlarged pelvic and retroperitoneal lymph nodes, hydronephrosis, and osteoblastic metastasis in prostate carcinoma. MRI detects and localizes cancer when the prostate-specific antigen is persistently elevated despite negative biopsy. ${ }^{[5,11,12]}$

In our patient, plain CT KUB showed well-defined isodense circumferential thickening around the abdominal aorta below the level of superior mesenteric artery, suggestive of retroperitoneal fibrosis. MRI abdomen and pelvis showed the medial deviation of ureters, multiple bone metastases and enlarged prostate, features consistent with retroperitoneal fibrosis, and metastatic prostatic malignancy.

Retroperitoneal fibrosis can be associated with Crohn's disease, ulcerative colitis, and sclerosing cholangitis.

The goal of treatment in retroperitoneal fibrosis is to relieve symptoms and to restore renal function. Surgical intervention includes ureterolysis and ureteric stent placement. Medications such as corticosteroids and immunosuppressive agents are curative in up to two-thirds of patients. ${ }^{[13-15]}$

Treatment for localized prostate cancer is radical prostatectomy, radiation therapy, and androgen deprivation 
therapy. Metastatic cancer is rarely curable. Our patient was treated with diuretics, antihypertensives, corticosteroids, and other supportive treatment as he was not willing for any surgical management.

Prognosis in retroperitoneal fibrosis is excellent $(>90 \%)$. Median survival is 3-6 months in malignant fibrosis..$^{[3,15]}$ Careful follow-up is mandatory since recurrences are often asymptomatic. ${ }^{[8]}$ Five-year survival is more than $99 \%$ with local prostate cancer and 28\% with distant disease.

\section{Conclusion}

Retroperitoneal fibrosis is an unusual presentation of prostate carcinoma. Thus, a high index of suspicion is required to avoid prostate carcinoma being missed, delayed, or misdiagnosed as the patient may not have any prior history of prostate carcinoma, urinary symptoms, or prostate-specific antigen screening tests done before.

\section{Financial support and sponsorship}

Nil.

\section{Conflicts of interest}

There are no conflicts of interest.

\section{References}

1. Powell CR, Schwartz BF, Stoney RJ, Stoller ML. Gross hematuria secondary to renal vein hypertension from unilateral retroperitoneal fibrosis. Urology 2000;55:436.

2. Kottra JJ, Dunnick NR. Retroperitoneal fibrosis. Radiol Clin North Am 1996;34:1259-75.

3. Vivas I, Nicolás AI, Velázquez P, Elduayen B, Fernández-Villa T, Martínez-Cuesta A. Retroperitoneal fibrosis: Typical and atypical manifestations. Br J Radiol 2000;73:214-22.
4. Amis ES Jr. Retroperitoneal fibrosis. AJR Am J Roentgenol 1991;157:321-9.

5. Bonekamp D, Jacobs MA, El-Khouli R, Stoianovici D, Macura KJ. Advancements in MR imaging of the prostate: From diagnosis to interventions. Radiographics 2011;31:677-703.

6. van Bommel EF. Retroperitoneal fibrosis. Neth J Med 2002;60:231-42.

7. Miller OF, Smith LJ, Ferrara EX, McAleer IM, Kaplan GW. Presentation of idiopathic retroperitoneal fibrosis in the pediatric population. J Pediatr Surg 2003;38:1685-8.

8. Monev S. Idiopathic retroperitoneal fibrosis: Prompt diagnosis preserves organ function. Cleveland Clin J Med 2002;69:160-6.

9. Loeb S, Carter HB. Early detection, diagnosis, and staging of prostate cancer. In: Wein AJ, Kavoussi LR, Novick AC, Partin AW, Peters CA, editors. Campbell-Walsh Urology. $10^{\text {th }}$ ed. Ch. 99. Philadelphia, PA: Elsevier Saunders; 2011.

10. Caiafa RO, Vinuesa AS, Izquierdo RS, Brufau BP, Ayuso Colella JR, Molina CN. Retroperitoneal fibrosis: Role of imaging in diagnosis and follow-up. Radiographics 2013;33:535-52.

11. Verma S, Rajesh A. A clinically relevant approach to imaging prostate cancer: Review. AJR Am J Roentgenol 2011;196 3 Suppl:S1-10.

12. Verma S, Turkbey B, Muradyan N, Rajesh A, Cornud F, Haider MA, et al. Overview of dynamic contrast-enhanced MRI in prostate cancer diagnosis and management. AJR Am J Roentgenol 2012;198:1277-88.

13. Ilie CP, Pemberton RJ, Tolley DA. Idiopathic retroperitoneal fibrosis: The case for nonsurgical treatment. BJU Int 2006;98:137-40.

14. Tomita Y, Morishita H, Saitoh R. Successful treatment of idiopathic retroperitoneal fibrosis with steroid administration in a patient with a positive gallium scan. Clin Nucl Med 1993;18:1042-4.

15. Kermani TA, Crowson CS, Achenbach SJ, Luthra HS. Idiopathic retroperitoneal fibrosis: A retrospective review of clinical presentation, treatment, and outcomes. Mayo Clin Proc 2011;86:297-303. 